\title{
Handwritten Character Recognition in English: A Survey
}

\author{
Monica Patel ${ }^{1}$, Shital P. Thakkar ${ }^{2}$ \\ Department of Electronics and Communication, Dharmsinh Desai University, Nadiad, Gujarat, India ${ }^{1}$
}

Associate Professor, Dept of Electronics and Communication, Dharmsinh Desai University, Nadiad, Gujarat, India ${ }^{2}$

\begin{abstract}
This paper presents a comprehensive review of Handwritten Character Recognition (HCR) in English language.The handwritten character recognition has been applied in variety of applications like Banking sectors, Health care industries and many such organizations where handwritten documents are dealt with. Handwritten Character Recognition is the process of conversion of handwritten text into machine readable form. For handwritten characters there are difficulties like it differs from one writer to another, even when same person writes same character there is difference in shape, size and position of character. Latest research in this area has used different types of method, classifiers and features to reduce the complexity of recognizing handwritten text.
\end{abstract}

Keywords: Handwritten database, features extraction, classifiers, HCR system.

\section{INTRODUCTION}

Handwritten character recognition (HCR) is the process of conversion of handwritten text into machine readable form. The major problem in handwritten character recognition (HCR) system is the variation of the handwriting styles, which can be completely different for different writers. The objective of handwritten character recognition system is to implement user friendly computer assisted character representation that will allow successful extraction of characters from handwritten documents and to digitalize and translate the handwritten text into machine readable text.

Handwritten character Recognition system is divided into two categories

- $\quad$ Recognization based segmentation Approach Character classification and segmentation are performed simultaneously by using appropriate learning method - $\quad$ Mixed Approach This system consist of combination of above methods

In this paper we present concise survey of available HCR for English language. HCR techniques are discussed with their strength and weaknesses. Different types of features are extracted and different types of classifiers are used to classify the input characters. The current study is focused on investigation of possible techniques to develop an offline HCR system for English language for both separate characters and cursive words.

\section{Motivation}

- $\quad$ On-line character recognition.

It is system in which recognition is performed when characters are under creation.

- $\quad$ Off-line character recognition.

It is system in which first handwritten documents are generated, scanned, stored in computer and than they are recognized.

Handwritten Character Recognition System consists of following stages:

1) Pre-processing.

2) Segmentation.

3) Feature extraction.

4) Training and recognition.

5) Post processing.

There are four methods of cursive handwritten word recognition.

- Holistic Approach

It is method in which entire word is recognized without splitting them by extracting features of entire word.

- $\quad$ Segmentation based Approach

Characters are segmented from word.

\section{STRUCTURE OF HCR SYSTEM}

The block diagram of the handwritten character recognition system is shown in figure 1 . 


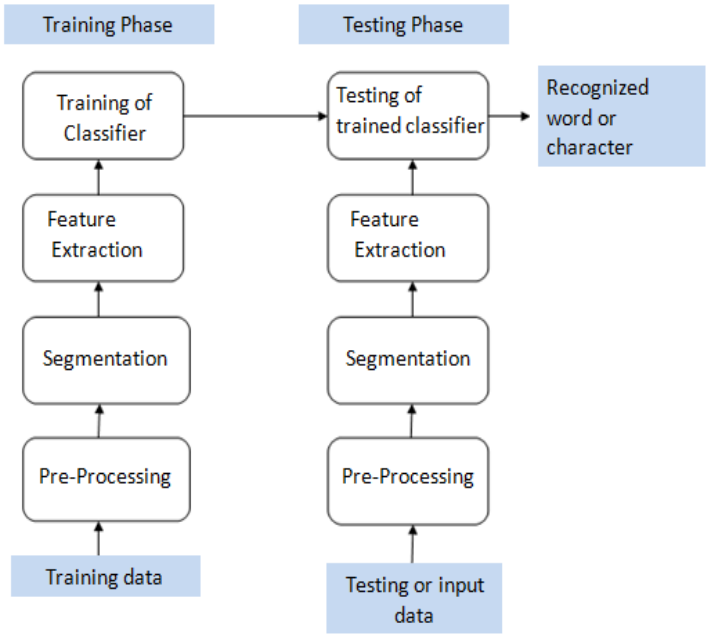

Fig 1: Block diagram of HCR System

The collected databases are divided into two parts Training data and testing data. Training data are used to train the system and this trained system are than used to recognize test data.

\section{A. Pre-processing}

The pre-processing is a sequence of operations performed on the scanned input image. It essentially enhances the image making it suitable for further processing. The various tasks performed on the image in pre-processing stage are noise removal, binarization, skew correction etc

\section{- Noise removal}

It is a process of removing noise from scanned image by using appropriate filter for example smoothing linear filter, order statistic filter etc. Smoothing is used for blurring and reducing noise, and removal of small details from the image extracting large objects.

\section{- Binarization}

It converts a gray scale image into a binary image using global thresholding technique like otsu's method of thresholding. Otsu's provide optimum value of threshold.

\section{- $\quad$ Skew correction}

It is removal of skew in scanned document for its proper further segmentation. It is not necessary that handwritten documents are perfectly horizontally aligned thus skew correction methods are required to be performed.

For example projection profile analysis, Hough transforms, nearest neighbour clustering, cross-correlation, piece-wise covering by parallelogram etc

\section{$B$ Segmentation}

In the segmentation stage, an image is decomposed into sub-images of individual character. Segmentation includes:

- line segmentation which is separation of line from paragraph,

- Word segmentation which is separation of word from line.

- Character segmentation which is separation of character from words.
Character segmentation is performed if segmentation based method is adopted for cursive word recognition, for holistic method character segmentation is not performed.

$C$ Feature Extraction

In this stage, the features of the characters that are essential for classifying them at recognition stage are extracted. This is an important stage as its successful operation improves the recognition rate and reduces the misclassification. Features like binary features, directional features etc are extracted and feature vector is created. Feature extraction methods falls among these categories.

- $\quad$ statistical features

It is based on the probability theory and hypothesis. Statistical distribution of pixels of an image takes care of variations in writing styles. Statistical features are derived from the statistical distribution of points. For example Projections histogram, crossings, distances, zoning etc.

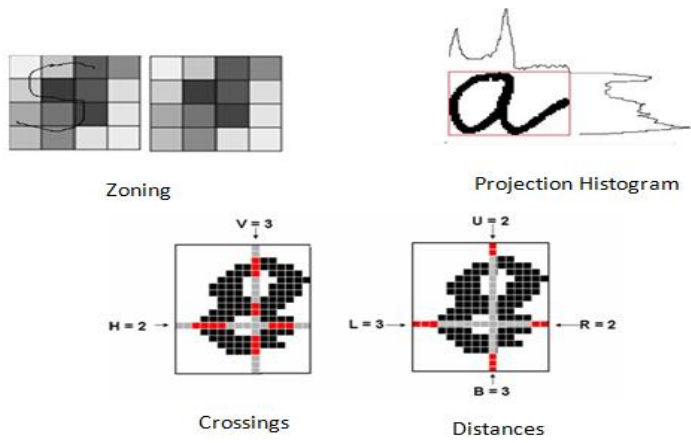

Figure 2: Statistical Features

- $\quad$ structural features

Structural features give information about structure of the image. Structural features describe the geometrical and topological properties of character, like crossing points,

Branches, loops, stroke length, stroke width, up, down, left and right projection profiles etc.
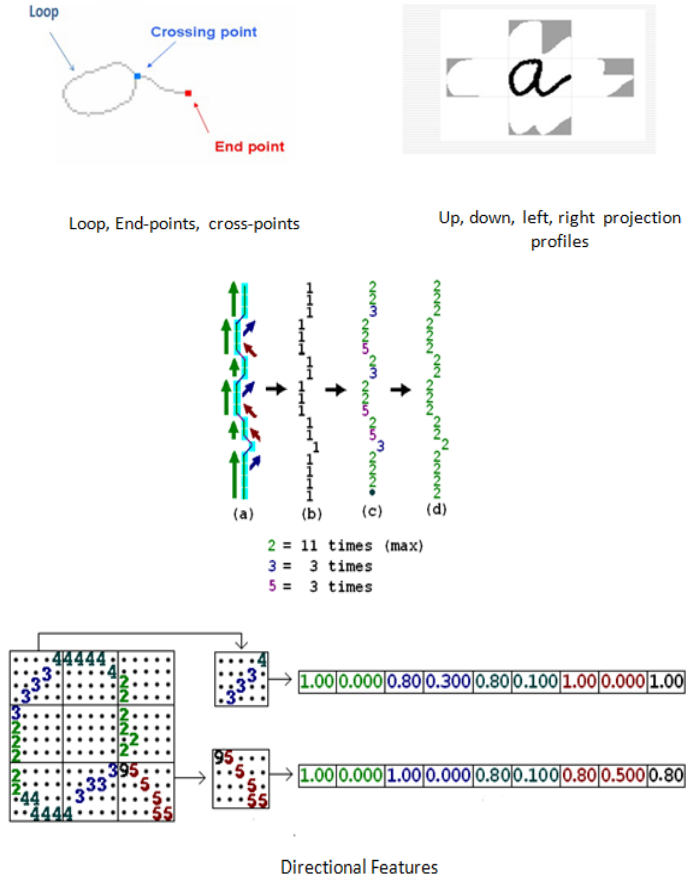

Figure 3: Structural features 
- Global transformation feature

Global transformation based features give well Classifiers are not used for recognization of words, rating representation of image shape. It is spatial domain to is given to each segment which are separated by prefrequency domain translation of image. This features segmentation using letter hypothesis and they are stores information contained in whole image in few recognized based on maximum value of rating coefficients, thus it performs energy compactness. Various types of global transformation based features are: Discrete Fourier Transform, Discrete Cosine Transform, Discrete Wavelet Transform etc.

\section{D-Classification}

The classification stage is the decision making part of a recognition system and it uses the features extracted in the previous stage. The feature vector is denoted as $\mathrm{X}$ where $\mathrm{X}=(\mathrm{f} 1, \mathrm{f} 2, \ldots . ., \mathrm{fd})$ where $\mathrm{f}$ denotes features and $\mathrm{d}$ is the no. features extracted from character. Based on the comparison of feature vector characters are efficiently classified into appropriate class and recognized.

Classifiers are based on two types of learning methods.

\section{- $\quad$ Supervised learning}

In supervised learning training data with correct detail of class is applied to train a model. This model is used to test data for proper classification. Training data includes both the input and the desired results. The model undergoes learning process and based on this learning it classifies test data.

For example: SVM, HMM etc.

\section{- Unsupervised learning}

In unsupervised learning model is not provided with training data. It does not require learning. The model classifies test data based on statistical properties and by their spatial grouping and considering their nearest neighbour.

For example: Clustering, $\mathrm{k}$ means etc

\section{E-Post-Processing}

In this stage accuracy of recognition is further increased by connecting dictionary to the system in order to perform Syntax analysis, semantic analysis kind of higher level concepts, which is applied to check the recognized character. This stage is not compulsory in HCR system.

\section{RELATED WORK}

Recognition accuracy of the image depends on the sensitivity of the selected features and type of classifier used. Hence, number of feature extraction and classification methods can be found in the literature.

Following paper perform handwritten character recognition of cursive words.

Radmilo M. Bozinovic and Sargur N. Srihari (1989)[1] This paper use Holistic method for cursive word recognization. The approach used here is to represent word through various stages of transformation like points, contours, features, letter and word. A unique feature vector is generated from the image using statistical dependences between letter and feature; partially computed words are recognized by comparing with lexicon. Lexicon includes
H. Bunke, M. Roth and E. G. Schukat-Talamazzini (1995) recognition. They extract features from the skeleton of word. The feature vector is generated from the edge information of words which includes location of edge relative to four reference line, its curvature, degree of nodes incident to the edge etc. 10-dimensional feature vector is generated.HMM for each letter of alphabets is built and by concatenation of this HMMs, HMM for each dictionary word is built. Limited sized dictionary is used. HMM is trained using Baum-Welch algorithm and recognization is performed using Viterbi algorithm.

Nafiz Arica (1998) [3]. The author had performed recognization both cursive and isolated handwritten characters using HMM. Hybrid method is used to maximize the superiority of HMM. For recognization of characters features used are medians of black run in each scan line. Character image is scanned in four different directions for extracting feature. Medians in each direction represent a sparse directional skeleton of the character. The discrete density left to right HMM is used for recognization. For cursive he used recognization based segmentation approach. Features are fed to the higher order HMM and finally segmentation path are confirmed. Correct segmentation points are found using graph search method in which shortest path with minimum cost. The probability of observation sequence of HMM are used for recognization.

Yong Haw Tay, Pierre-Michel Lallican, Marzuki Khalid, Christian Viard-Gaudin, Stefan Knerr (2001) [4]. This paper recognizes handwritten cursive words using recognization based segmentation method. This paper gives the comparison between two methods. The first recognization system uses combination of Neural Network and HMM (Hidden Markov Model) for recognization. In second method discrete HMM is used. It first method Presegmentation of word is performed using segmentation graph. Neural network calculates the probability for each letter hypothesis in graph and then HMM computes likelihood for each word in lexicon by adding the probability along each possible path in graph. In second method 140 geometric features are extracted from each segment which is separated by pre-segmentation. This features by vector quantization (VQ) converted to single symbol and finally by calculating the likelihood for each word in lexicon word is recognized.

Anshul Gupta, Manisha Srivastava and Chitralekha Mahanta. (2011) [5] .In this paper author used segmentation based approach for cursive word recognization. In this method cursive words are first segmented into individual characters, which are than [2]. This paper use holistic method for cursive word 
recognized and merged to produce meaningful word by comparing with dictionary. The dictionary used in this paper consists of 26 words. Thus scope of this paper is limited to 26 words.

Following paper perform handwritten character recognition of separate characters.

J. Pradeep, E. Srinivasan and S. Himavathi (2012) [6] has designed Neural Network based recognition system. They used different neural network (NN) topologies- back propagation neural network, nearest neighbour network and radial basis function network for same training dataset. They compared the performance of each network and optimized the number of neurons in hidden layer which is not dependent on initial value and concluded that combination of standard feature extraction technique with feed forward back propagation

D. K. Patel, T. Som and M. K Singh (2012) [7] deals with the handwritten English character recognition using multiresolution technique with Discrete Wavelet Transform (DWT) and Euclidean Distance Metric (EDM). Distances from unknown input pattern vector to all the mean vectors are calculated by EDM. Minimum distance determines the class membership of input pattern vector. EDM gives a recognition accuracy of $90.77 \%$. In case of misclassification, the learning rule through ANN improves the recognition accuracy to $95.38 \%$ by comparing scores and then product of generated recognition scores with Euclidean distances has further improves the recognition accuracy to $98.46 \%$.

M. Blumenstein, B. Verma and H. Basli (2003) [8]. This research describes neural network-based techniques for segmented character recognition. Two neural architectures along with two different feature extraction techniques were investigated. Directional and Transition features are used and compared by using Back-Propagation (BP) and Radial Basis Function (RBF) networks classifiers. The size of feature vector is 100 in case of transition feature and 81 for directional feature. Experiment was performed by using the CAS dataset, the BP (Back propagation) and $\mathrm{RBF}$ (Radial basis function) algorithm using two feature extraction techniques for both lower case and upper case characters, similarly for BAC database. Directional features using neural network perform better than transition features.

Sumedha B. Hallale, Geeta D. Salunke (2013) [9]. In this paper comparison between conventional and directional feature extraction method is done. Twelve directional features are used for recognition of alphabets and numerals. In order to extract directional feature gradient feature of each pixel are extracted the gradient values are mapped onto 12 direction values to the angle span of 30 degree between any two adjacent direction values. Feature vector of each class is obtained by taking mean of feature matrix of each class. The similarity between testing feature vector and feature vector of all the classes is calculated, testing image belongs to the class which has the highest similarity.
Amit Choudhary, Rahul Rishi and Savita Ahlawat (2013) [10]. In this paper handwritten character recognition of lowercase English alphabets is performed by using binarized pixels of the image as features and multilayer back-propagation neural network as classifier. The character image is binarized, filtered and resized to $15 \times 12$, thus feature vector of size 180 is created of each character which is given to neural network for its training. MSE (mean square error) is used as cost function. The use of binarization features with back-propagation neural network classifier gives classification accuracy of $85.62 \%$. It has simplicity of features as direct pixel values are taken

Rafael M. O. Cruz, George D. C. Cavalcanti and Tsang Ing Ren (2010) [11].In this paper recognization separate handwritten cursive characters is performed. Here different features are extracted among them two featuresmodified edge map and multiple zoning are proposed by authors. Total nine features are extracted and drawback of each feature each overcome by other. Each features are individually given as input to nine multilayer perceptron network and output of all this classifier are combined with each other by different rule like sum rule, product rule, max rule, mean rule etc among them trained MLP combiner gives maximum result. Among proposed features modified edge map feature gives highest result.

Table 1 has concise details of all papers that have be read by us.

TABLE 1

Literature Survey

\begin{tabular}{|c|c|c|c|}
\hline $\begin{array}{l}\text { Author \& } \\
\text { Year }\end{array}$ & $\begin{array}{c}\text { Classifie } \\
\mathbf{r}\end{array}$ & Features & Accuracy \\
\hline $\begin{array}{l}\text { R. } \\
\text { M.Bozinovic, } \\
\text { S. N. Srihari. } \\
(1989)\end{array}$ & $\begin{array}{l}\text { Word } \\
\text { formation } \\
\text { using } \\
\text { letter } \\
\text { hypothesi } \\
\mathrm{s}\end{array}$ & $\begin{array}{l}\text { Contour } \\
\text { tracing, event } \\
\text { construction, } \\
\text { letter } \\
\text { hypothesis } \\
\text { and word } \\
\text { hypothesis }\end{array}$ & $\begin{array}{l}77 \% \\
\text { For dictionary of } \\
130 \text { words } \\
\text { 66-training } \\
\text { 64-different test set } \\
\text { For cursive }\end{array}$ \\
\hline $\begin{array}{l}\text { H. Bunke, } \\
\text { M. Roth, } \\
\text { E. G. } \\
\text { Schukat- } \\
\text { Talamazzini. } \\
\text { (1995) }\end{array}$ & HMM & $\begin{array}{l}\text { Location, } \\
\text { curvature of } \\
\text { edge and } \\
\text { percentage of } \\
\text { pixels lying } \\
\text { on the edge }\end{array}$ & $\begin{array}{l}98 \% \\
\text { For dictionary of } \\
150 \text { words } \\
\text { 2250-training } \\
\text { 750-testing } \\
\text { On ruled paper } \\
\quad \text { For cursive }\end{array}$ \\
\hline $\begin{array}{l}\text { Nafiz Arica } \\
\text { (1998) }\end{array}$ & HMM & $\begin{array}{l}\text { medians of } \\
\text { black run in } \\
\text { vertical } \\
\text {,horizontal } \\
\text { and diagonal } \\
\text { scan lines for } \\
\text { getting } \\
\text { directional } \\
\text { skeleton }\end{array}$ & $\begin{array}{l}65 \% \text { 240 } \\
\text { cursive words for } \\
\text { names on the bank } \\
\text { checks, } 19 \text { distinct } \\
\text { characters } \\
\text { segmented } \\
\text { manually, } 20 \\
\text { samples for each } \\
\text { class used for } \\
\text { HMM training, } \\
\text { 300- testing } \\
\text { For cursive }\end{array}$ \\
\hline $\begin{array}{l}\text { Y. H. Tay, } \\
\text { P.M. Lallican, } \\
\text { M. Khalid, } \\
\text { Christian }\end{array}$ & $\begin{array}{l}\mathrm{HMM}+\mathrm{N} \\
\mathrm{N}\end{array}$ & $\begin{array}{l}140 \\
\text { geometrical } \\
\text { features of } \\
\text { each pre }\end{array}$ & $\begin{array}{c}96.1 \% \\
196 \text { word lexicon } \\
\text { 24177-training } \\
\text { 12219-testing }\end{array}$ \\
\hline
\end{tabular}


International Journal of Advanced Research in Computer and Communication Engineering Vol. 4, Issue 2, February 2015

\begin{tabular}{|c|c|c|c|}
\hline $\begin{array}{l}\text { Gaudin, } \\
\text { Stefan Knerr. } \\
(2001)\end{array}$ & & $\begin{array}{l}\text { segmented } \\
\text { frames }\end{array}$ & For cursive \\
\hline $\begin{array}{l}\text { A. Gupta, M. } \\
\text { Srivastava, } \\
\text { C. Mahanta. } \\
\text { (2011) }\end{array}$ & $\begin{array}{l}\mathrm{NN}+ \\
\mathrm{SVM}\end{array}$ & $\begin{array}{l}\text { Fourier } \\
\text { Descriptors }\end{array}$ & $\begin{array}{l}62.93 \% \text { on test data } \\
26 \text { word lexicon } \\
260 \text { words each } 10 \\
\text { times } \\
26 \text {-testing } \\
\text { For cursive }\end{array}$ \\
\hline $\begin{array}{l}\text { Author \& } \\
\text { Year }\end{array}$ & $\begin{array}{c}\text { Classifie } \\
\mathbf{r}\end{array}$ & Features & Accuracy \\
\hline $\begin{array}{l}\text { J. Pradeep, E. } \\
\text { Srinivasan } \\
\text { S. Himavathi } \\
(2012)\end{array}$ & $\mathrm{NN}$ & $\begin{array}{lr}\text { character } & \\
\text { resized into } \\
30 X 20 \text { pixels } \\
\text { taken } \\
\text { feature }\end{array}$ & $\begin{array}{l}94.15 \% \\
200 \text { database of } \\
\text { each } 26 \text { characters } \\
\text { Capital characters }\end{array}$ \\
\hline $\begin{array}{l}\text { D. K. Patel, } \\
\text { T. Som, } \\
\text { M. K Singh } \\
(2012)\end{array}$ & ANN & $\begin{array}{l}\text { Discrete } \\
\text { Wavelet } \\
\text { Transform } \\
\text { (DWT) }\end{array}$ & $\begin{array}{l}98.46 \% \\
100 \text { samples of each } \\
\text { character for } \\
\text { training, } 50 \text { samples } \\
\text { for testing } \\
\text { Capital characters }\end{array}$ \\
\hline $\begin{array}{l}\text { M. } \\
\text { Blumenstein, } \\
\text { B. Verma, H. } \\
\text { Basli (2003) }\end{array}$ & $\begin{array}{l}\text { BP and } \\
\text { RBF } \\
\text { networks }\end{array}$ & $\begin{array}{l}\text { Directional } \\
\text { and Transition } \\
\text { features }\end{array}$ & $\begin{array}{l}85.48 \% \text { for } \\
\text { uppercase and } \\
70.63 \% \text { for } \\
\text { lowercase, CAS and } \\
\text { BAC database } \\
\text { Small characters }\end{array}$ \\
\hline $\begin{array}{l}\text { S. B. Hallale, } \\
\text { G. D. Salunke } \\
(2013)\end{array}$ & $\begin{array}{l}\text { direction } \\
\text { al Pattern } \\
\text { matching }\end{array}$ & $\begin{array}{l}\text { Twelve } \\
\text { directional } \\
\text { features }\end{array}$ & $\begin{array}{l}88.29 \% \\
500 \text { training images } \\
\text { and } 200 \text { testing } \\
\text { images } \\
\text { Capital characters } \\
\text { and numerals }\end{array}$ \\
\hline $\begin{array}{l}\text { A. } \\
\text { Choudhary, } \\
\text { R. Rishi, } \\
\text { S. Ahlawat } \\
\text { (2013) }\end{array}$ & $\mathrm{NN}$ & $\begin{array}{l}\text { Character } \\
\text { image resized } \\
\text { to } 15 \times 12 \text { size, } \\
\text { feature vector } \\
\text { of size } 180 \text { is } \\
\text { created }\end{array}$ & $\begin{array}{l}85.62 \% . \\
\text { Database from } 10 \\
\text { peoples } 5 \text { samples } \\
\text { from each thus total } \\
10 \times 5 \times 26=1300 \\
\text { character image } \\
\text { small characters }\end{array}$ \\
\hline $\begin{array}{l}\text { Rafael M. O. } \\
\text { Cruz, George } \\
\text { D. C. } \\
\text { Cavalcanti } \\
\text { and Tsang Ing } \\
\text { Ren (2010). }\end{array}$ & MLP & $\begin{array}{l}\text { Modified } \\
\text { Edge Maps } \\
\text { and Multi } \\
\text { zoning. }\end{array}$ & $\begin{array}{l}91.39 \% \text { for } \\
\text { uppercase } \\
88.45 \% \text { for } \\
\text { lowercase } \\
\text { C-Cube Database } \\
\text { Separate Cursive } \\
\text { Characters }\end{array}$ \\
\hline
\end{tabular}

\section{CONCLUSION}

Immense work and research has been done in the handwritten separate character recognition. But so far $100 \%$ accuracy is not achieved which gives scope of further work in this direction. Separate characters give good accuracy but word recognition is affected by different writing style. Holistic method eliminates the complicate segmentation but they use limited vocabulary. Segmentation based method due to its complexity acquire less accuracy. Good accuracy is observed in the classifier where scope of words is limited to fix numbers as it has to deal with limited number of variation.

\section{REFERENCES}

[1] Radmilo M.Bozinovic and Sargur N. Srihari, "Off-line Cursive Script word recognition", IEEE Transactions On Pattern Analysis and Machine Intelligence, Vol.11. No. 1, January 1989.

[2] H. Bunke, M. Roth and E.G.Schukat-Talamazzini, "Offline Cursive Handwriting Recognition Using Hidden Markov Models", Pattern Recognition, Vol. 28, No. 9, 1995 Elsevier Science Ltd.

[3] Nafiz Arica, "An Off-line Character Recognition System for free style Handwriting", a thesis submitted to the graduate school of natural and applied sciences of the middle east technical university, 1998.

[4] Yong Haw Tay, Pierre-Michel Lallican, Marzuki Khalid, Christian Viard-Gaudin, Stefan Knerr," An Offline Cursive Handwritten Word Recognition System", IEEE Catalogue No. 01 CH37239 2001.

[5] Anshul Gupta, Manisha Srivastava and Chitralekha Mahanta," Offline Handwritten Character Recognition International Conference on Computer Applications and Industrial Electronics (ICCAIE), 2011.

[6] J. Pradeep, E. Srinivasan and S. Himavathi,"Neural Network Based Recognition System Integrating Feature Extraction and Classification for English Handwritten", International Journal of Engineering (IJE) Transactions B: Applications Vol. 25, No. 2, (May 2012) 99-106

[7] D. K. Patel, T. Som and M. K Singh,” Improving the Recognition of Handwritten Characters using Neural Network through Multiresolution Technique And Euclidean Distance Metric", International Journal of Computer Applications (0975 - 8887) Volume 45- No.6 May 2012.

[8] M. Blumenstein, B. Verma and H. Basli," A Novel Feature Extraction Technique for the Recognition of Segmented Handwritten Characters", Proceedings of the Seventh International Conference on Document Analysis and Recognition (ICDAR'03) 0-7695-1960-1/03 \$17.00 @ 2003 IEEE

[9] Sumedha B. Hallale, Geeta D. Salunke, "Twelve Directional Feature Extraction for Handwritten English Character Recognition", International Journal of Recent Technology and Engineering (IJRTE)ISSN:2277-3878, Volume-2, Issue-2, May 2013.

[10] Amit Choudhary, Rahul Rishi and Savita Ahlawat, "Off-Line Handwritten Character Recognition using Features Extracted from Binarization Technique", 2212-6716 @ 2013 American Applied Science Research Institute doi:10.1016/j.aasri.2013.10.045

[11] Rafael M. O. Cruz, George D. C. Cavalcanti and Tsang Ing Ren," An Ensemble classifier for offline Cursive character recognition using multiple features Extraction technique", 978-1-4244-81262/10/\$26.00 @2010 IEEE

\section{BIOGRAPHIES}

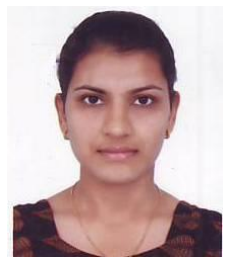

Monica Patel received bachelor of Engineering degree in Electronics and Communication Engineering in 2013 from Gujarat Technological University, Ahmedabad, Gujarat, India. She is pursuing Master of Technology in Electronics and communication Systems from Dharmsinh Desai University, Nadiad, Gujarat, India. Her area of interest is, Image processing.

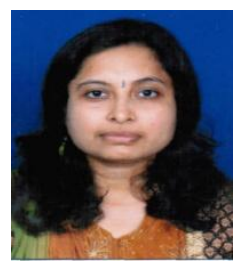

Shital Thakkar obtained her Master's degree in Electronics \& Communication Systems Engineering from Dharmsinh Desai University, Nadiad, India. She has experience of one year as an Apprentice at Space Application Centre (ISRO), Ahmedabad. She joined as a Lecturer in Electronics \& Communication Department, Dharmsinh Desai University, Nadiad, Gujarat, India, where she is currently working as an Associate Professor since 2007. Her research areas include Image Processing and Signal Processing. 
International Journal of Advanced Research in Computer and Communication Engineering Vol. 4, Issue 2, February 2015 